This is a preprint of an article accepted for publication in the Journal of Combinatorial Designs (C) 2010 (copyright owner as specified in the journal). 


\title{
Some rigid Steiner 5-designs
}

\author{
M. J. Grannell \\ Department of Mathematics and Statistics \\ The Open University, Walton Hall \\ Milton Keynes MK7 6AA \\ UNITED KINGDOM \\ (m.j.grannell@open.ac.uk)
}

January 8, 2010

\begin{abstract}
Hitherto, all known non-trivial Steiner systems $\mathrm{S}(5, k, v)$ have, as a group of automorphisms, either $\operatorname{PSL}(2, v-1)$ or $\operatorname{PGL}\left(2, \frac{v-2}{2}\right) \times C_{2}$. In this paper, systems $\mathrm{S}(5,6,72), \mathrm{S}(5,6,84)$ and $\mathrm{S}(5,6,108)$ are constructed that have only the trivial automorphism group.
\end{abstract}

\section{AMS classification: \\ 05B05.}

\section{Keywords:}

Steiner system; Steiner 5-design. 


\section{Introduction}

A Steiner system $\mathrm{S}(t, k, v)$, also called a Steiner $t$-design, is a pair $(V, \mathcal{B})$ where $V$ is a set of cardinality $v$ (the points) and $\mathcal{B}$ is a set of $k$-element subsets of $V$ (the blocks) which has the property that each $t$-element subset of $V$ is a subset of precisely one block. As is usual, we exclude trivial systems by requiring that $1<t<k<v$. An automorphism of a system $\mathrm{S}(t, k, v)=(V, \mathcal{B})$ is a mapping $\phi$ defined on $V$ that preserves $\mathcal{B}$. A system is said to be rigid, or to have the trivial automorphism group, if the only automorphism is the identity mapping.

Amongst possible parameters $(t, k)$, the smallest case of interest is in Steiner systems $\mathrm{S}(2,3, v)$, generally known as Steiner triple systems. A necessary and sufficient condition for the existence of these is that $v \equiv 1$ or $3(\bmod 6)[12]$. Many constructions are known for Steiner triple systems and an extensive survey is given in [4]. Systems $\mathrm{S}(3,4, v)$ are generally known as Steiner quadruple systems, and a necessary and sufficient condition for the existence of these is that $v \equiv 2$ or $4(\bmod 6)$, see for example [10]. Systems $\mathrm{S}(2, k, v)$ with $k>3$ and $\mathrm{S}(3, k, v)$ with $k>4$ have also been investigated, and results are surveyed in $[1,11]$.

By contrast, for $t=4$ and 5 , only a finite number of Steiner systems $\mathrm{S}(t, k, v)$ are known, and no systems are known with $t>5$. Systems $\mathrm{S}(5, k, v)$ are only known for $(k, v)=(6,12),(8,24),(6,24),(7,28),(6,36),(6,48),(6,72),(6,84)$, $(6,108),(6,132),(6,168)$ and $(6,244)$. All the known systems with these parameters, apart from $\mathrm{S}(5,6,36)$, have $\operatorname{PSL}(2, v-1)$ as an automorphism group, and the only known $\mathrm{S}(5,6,36)$ has $\mathrm{PGL}(2,17) \times C_{2}$. The systems $\mathrm{S}(5,6,12)$ and $\mathrm{S}(5,8,24)$, which are unique up to isomorphism, have the larger groups $M_{12}$ and $M_{24}$ respectively as their full automorphism groups, and all known $\mathrm{S}(5,6,244) \mathrm{s}$ have $\mathrm{P} \Sigma \mathrm{L}(2,243)$. For references, see $[2,3,5,8,9,13,14]$.

Given an $\mathrm{S}(t, k, v)=(V, \mathcal{B})$, a derived system $\mathrm{S}(t-1, k-1, v-1)$ may be formed as follows. First select any point $x \in V$, then remove $x$ from all the blocks that contain it. The resulting set $\mathcal{B}^{\prime}$ of $(k-1)$-tuples provides the blocks of the $\mathrm{S}(t-1, k-1, v-1)$ on the point set $V^{\prime}=V \backslash\{x\}$. By repeating this process, non-trivial derived Steiner systems $\mathrm{S}(t-i, k-i, v-i)$ may be formed for $1 \leq i \leq t-2$. All known systems $\mathrm{S}(4, k, v)$ are derived systems. In a sense therefore, all known Steiner systems $\mathrm{S}(t, k, v)$ with $t \geq 4$ are associated with an automorphism group PSL or PGL.

There are classical constructions for the systems $\mathrm{S}(5,6,12)$ and $\mathrm{S}(5,8,24)$ but, starting with the 1976 paper of Denniston [5], all remaining $\mathrm{S}(5, k, v)$ systems have been constructed by taking a suitable group $G$ (such as PSL $(2, v-1)$ ) acting on a set of $v$ points, computing the orbits of $k$-tuples under $G$, and then searching for a suitable collection of these orbits to form the system. It is therefore of some interest to consider whether the involvement of a PSL or PGL group as an automorphism group is intrinsic to systems $\mathrm{S}(5, k, v)$, or whether the prevalence of these groups as automorphisms of all the systems found in the past 30 years is due to the fact that their properties make the construction of the design so much easier than it would otherwise be. The particularly useful property of $\operatorname{PSL}(2, q)$, for $q$ a prime power with $q \equiv 3(\bmod 4)$, is the triple 
homogeneity of its action on a set of $v=q+1$ points. The group $\operatorname{PSL}(2, q)$ may be represented as the following set of mappings $\phi$ acting on the point set $V=$ $\mathrm{GF}(q) \cup\{\infty\}:$

$$
\left\{\phi: z \mapsto \frac{a z+b}{c z+d}, \quad \begin{array}{l}
\text { where } a, b, c, d \in \operatorname{GF}(q) \text { and } a d-b c \text { is } \\
\text { a non-zero quadratic residue in } \operatorname{GF}(q) .
\end{array}\right\}
$$

Triple homogeneity means that any unordered triple from $V$ can be mapped to any other such triple by an element of the group. As a consequence, if an $\mathrm{S}(5, k, q+1)$ is formed from orbits under $\operatorname{PSL}(2, q)$ with $q \equiv 3(\bmod 4)$ as described, then all its derived $\mathrm{S}(2, k-3, v-3)$ systems are isomorphic. And to verify that a particular collection of $\operatorname{PSL}(2, q)$ orbits does indeed form an $\mathrm{S}(5, k, q+1)$ when $q \equiv 3(\bmod 4)$, it suffices to check that any one derived set of $(k-3)$-tuples (obtained by taking any three points $x, y, z$ and deleting these from the $k$-tuples in which they all lie together) forms an $\mathrm{S}(2, k-3, v-3)$. The simplification afforded by this property is substantial; instead of having to check that $\frac{v(v-1)(v-2)(v-3)(v-4)}{k(k-1)(k-2)(k-3)(k-4)} \quad k$-tuples cover each 5 -tuple of points from $V$

precisely once, one only has to check that $\frac{(v-3)(v-4)}{(k-3)(k-4)}(k-3)$-tuples cover each pair of points from $V^{\prime}=V \backslash\{x, y, z\}$ precisely once.

\section{Method}

In essence, the method is to take an existing $\mathrm{S}(5,6, v)$, say $S=(V, \mathcal{B})$, that has a set of blocks $B \subseteq \mathcal{B}$ which can be removed and replaced by an alternative set of blocks $B^{\prime}$ covering the same 5-tuples as $B$, and hence to form another $\mathrm{S}(5,6, v)$, say $S^{\prime}=\left(V, \mathcal{B}^{\prime}\right)$, where $\mathcal{B}^{\prime}=(\mathcal{B} \backslash B) \cup B^{\prime}$. The sets $B$ and $B^{\prime}$ must also have the property that the replacement of $B$ by $B^{\prime}$ yields a rigid system.

The pair $\left\{B, B^{\prime}\right\}$ is an example of a combinatorial trade, which may be defined more generally for any $\mathrm{S}(t, k, v)$. In the context of such systems, a trade is a pair $\left\{T, T^{\prime}\right\}$ where $T$ and $T^{\prime}$ are disjoint sets of $k$-tuples, taken from a point set $V$, that cover precisely the same $t$-tuples with multiplicity one. The common cardinality of $T$ and $T^{\prime}$ is called the volume of the trade and the set of points covered is called the foundation of the trade. For Steiner triple systems, the smallest trade, both by volume and by foundation, is the Pasch or quadrilateral trade. A Pasch configuration or quadrilateral has the form $P=\{a b c, a d e, b d f, c e f\}$ where $a, b, c, d, e, f$ are distinct points. (Here and subsequently when no confusion is likely, we omit set brackets and commas from blocks such as $\{a, b, c\}$.) Whenever such a Pasch configuration occurs in a Steiner triple system, it can be replaced by the Pasch configuration $P^{\prime}=$ $\{f b c, f d e, b d a, c e a\}$ to form a new, although possibly isomorphic, Steiner triple system. The Pasch trade $\left\{P, P^{\prime}\right\}$ has volume 4 and foundation $\{a, b, c, d, e, f\}$. We will write $P=(a, b, c, d, e, f)$ to mean that the four blocks of $P$ are as stated, so that $P^{\prime}=(f, b, c, d, e, a)$.

By analogy, in the context of $\mathrm{S}(5,6, v)$ systems, we define a hyperquadrilateral $H=(a, b, c, d, e, f, g, h, i, j, k, l)$ to consist of the following 326 -tuples on 12 distinct points. 


$\begin{array}{cccc}\text { abcdef, } & \text { abcdgh, } & \text { abcegi, } & \text { abcfhi, } \\ \text { ajkdef, } & \text { ajkdgh, ajkegi, } & \text { ajkfhi, } \\ \text { bjldef, } & \text { bjldgh, } & \text { bjlegi, } & \text { bjlfhi, } \\ \text { ckldef, } & \text { ckldgh, cklegi, } & \text { cklfhi, } \\ \text { abjdeg, } & \text { abjdfh, } & \text { abjefi, } & \text { abjghi, } \\ \text { ackdeg, } & \text { ackdfh, } & \text { ackefi, } & \text { ackghi, } \\ \text { bcldeg, } & \text { bcldfh, } & \text { bclefi, } & \text { bclghi, } \\ \text { jkldeg, } & \text { jkldfh, } & \text { jklefi, } & \text { jklghi. }\end{array}$

If $H^{\prime}$ is formed from $H$ by exchanging $a$ and $l$, so that $H^{\prime}=(l, b, c, d, e, f, g, h, i, j$, $k, a)$, then it is routine to verify that $\left\{H, H^{\prime}\right\}$ is a trade with volume 32 and foundation $\{a, b, c, d, e, f, g, h, i, j, k, l\}$.

Given a hyperquadrilateral $H$ in a Steiner system $\mathrm{S}(5,6, v)=S$, its signature in $S, \operatorname{sig}(H, S)$, is defined to be the vector $\left(x_{1}, x_{2}, \ldots, x_{n}\right)$ where $x_{i}$ is the number of blocks of $H$ that lie in precisely $i$ hyperquadrilaterals. Thus $\sum_{i=1}^{n} x_{i}=32$. In the computations described below we encounter no hyperquadrilaterals with a signature vector of length greater than three, so we will write all these vectors as vectors of length three. Note that $H$ has no blocks in common with any other hyperquadrilateral in $S$ if and only if $\operatorname{sig}(H, S)=(32,0,0)$.

Our first goal is to find an $\mathrm{S}(5,6, q+1)$ fixed by $\operatorname{PSL}(2, q)$ that contains some hyperquadrilaterals. Any such system will be formed from orbits of 6 -tuples under $\operatorname{PSL}(2, q)$. The order of $\operatorname{PSL}(2, q)$ is $(q+1) q(q-1) / 2$, and the number of blocks in an $\mathrm{S}(5,6, q+1)$ is $(q+1) q(q-1)(q-2)(q-3) / 6$ !. Hence if such a system can be formed entirely of full orbits, $(q-1)(q-3) / 360$ orbits are required, while a system containing shorter orbits requires proportionately more. In principle, we could take a random subset of 12 points from $G F(q) \cup\{\infty\}$, write down a hyperquadrilateral with this set as its foundation, and then apply $\operatorname{PSL}(2, q)$ to each of the 32 blocks in the hope that the resulting set of blocks either formed, or could be completed to form, an $\mathrm{S}(5,6, q+1)$. However, the chances of success in a random attempt of this nature seem very remote, since for reasonably small values of $q$, say $q \leq 83$, several of the blocks of the hyperquadrilateral would have to lie together in some orbits. Of course, such a random procedure might succeed for a much larger system such as an $\mathrm{S}(5,6,244)$, but there are different, and possibly greater, computational challenges dealing with systems of this size.

There are precisely three nonisomorphic $\mathrm{S}(5,6,24)$ systems fixed by $\operatorname{PSL}(2,23)[6]$, and a short computer search establishes that none of these contain any hyperquadrilaterals. There are 459 nonisomorphic $\mathrm{S}(5,6,48)$ systems fixed by PSL $(2,47)[7]$ and, again, a computer search establishes that none of these contains any hyperquadrilaterals. For $v=72$ and 84 there are respectively exactly 926299 and at least 348512 nonisomorphic $\mathrm{S}(5,6, v)$ systems fixed by $\operatorname{PSL}(2, v-1)$ [2]. Although it might be feasible to carry out an exhaustive search for hyperquadrilaterals in these systems, the number of nonisomorphic $\mathrm{S}(5,6,108)$ systems fixed by $\operatorname{PSL}(2,107)$ is likely to be substantially larger, and for all three cases a different approach was used. Namely, it was assumed that the hyperquadrilateral has a specific form fixed by an order four subgroup of $\operatorname{PSL}(2, v-1)$. 


\section{$3 v=72$}

Take the mappings on $\operatorname{GF}(71) \cup\{\infty\}$ given by $\alpha: z \mapsto-\frac{1}{z}$ and $\beta: z \mapsto \frac{z+4}{4 z-1}$. Since $-17=14^{2}$ in $\operatorname{GF}(71), G_{71}=\langle\alpha, \beta\rangle$ forms an order 4 subgroup of $\operatorname{PSL}(2,71)$. We consider possible hyperquadrilaterals $H=(a, b, c, d, e, f, g, h, i, j$, $k, l)$ where

$$
\begin{array}{lll}
l=\alpha(a), & f=\beta(a), & g=\alpha \beta(a), \\
k=\alpha(b), & e=\beta(b), & h=\alpha \beta(b), \\
j=\alpha(c), & d=\beta(c), & i=\alpha \beta(c) .
\end{array}
$$

The 32 blocks of such a hyperquadrilateral will lie in at most ten orbits under $\operatorname{PSL}(2,71)$ because $G_{71}$ partitions the 32 blocks into six orbits of length 4 and four orbits of length 2 as shown in Table 1. For example, $\alpha(\{a b c d e f\})=$ $\{j k l g h i\}$.

\{abcdef,jklghi\}, $\quad\{a b c d g h$, ckldef,abjghi,jklefi\}, $\{a b c e g i, b j l d e f, a c k g h i, j k l d f h\}, \quad\{a b c f h i, a j k d e f, b c l g h i, j k l d e g\}$, $\{a j k d g h, c k l f h i, a b j d e g, b c l e f i\}, \quad\{a j k e g i, b j l f h i$, ackdeg, bcldf $h\}$, $\{a j k f h i, b c l d e g\}$, $\{$ bjlegi, ackdf $h\}$,

$\{b j l d g h$, cklegi,abjdf $h$, ackefi $\}$, $\{$ ckldgh,abjefi\}.

Table 1. Orbits under $G_{71}$.

Given $a, b$ and $c$, any one of the resulting PSL orbits may be unsuitable for inclusion in an $\mathrm{S}(5,6,72)$ because it contains a repeated 5-tuple, or two of them may be incompatible for a similar reason. It turns out that, up to equivalence, there are just six choices for the triple $(a, b, c)$ which do not fall foul of one of these obstacles. One of these is given by $(a, b, c)=(13,17,44)$. The corresponding hyperquadrilateral is $H_{1}=(13,17,44,66,48,24,68,34,57,50,25,60)$. The blocks of $H_{1}$ lie in just five orbits under PSL $(2,71)$. Each of these orbits may be characterized by a starter taken as the numerically lowest block appearing in the orbit (with $\infty$ taken as the lowest value); these starters are $\{\infty, 0,1,2,17,39\},\{\infty, 0,1,3,11,59\},\{\infty, 0,1,4,13,64\},\{\infty, 0,1,4,42,65\}$ and $\{\infty, 0,1,6,41,64\}$. The first of these gives a full orbit, the second, third and fourth give half orbits, and the last gives a sixth orbit.

Next, we attempt to form an $\mathrm{S}(5,6,72)$ as a union of orbits under $\operatorname{PSL}(2,71)$ that includes the five orbits generating $H_{1}$. By an exhaustive computer search, just two solutions were obtained. One of these has the starters (with $\infty, 0,1$ suppressed for brevity) shown in Table 2 .

$$
\begin{array}{lllll}
\{2,17,39\}, & \{3,11,59\}, & \{4,13,64\}, & \{4,42,65\}, & \{6,41,64\}, \\
\{2,3,27\}, & \{2,5,19\}, & \{2,6,58\}, & \{2,7,55\}, & \{2,9,16\}, \\
\{2,12,51\}, & \{2,21,40\}, & \{3,4,32\}, & \{3,7,26\}, & \{3,12,52\}, \\
\{3,14,18\}, & \{3,16,19\}, & \{3,22,30\}, & \{3,31,57\}, & \{4,9,22\}, \\
\{4,17,57\}, & \{4,26,61\}, & \{4,29,53\}, & \{6,14,31\} . &
\end{array}
$$

Table 2. Starters for an $\mathrm{S}(5,6,72)$. 
We will refer to the above system as $S$; it contains an orbit of hyperquadrilaterals under $\operatorname{PSL}(2,71)$. In particular, it contains $H_{1}$ and $H_{2}=(14,18,45,67,49,25$, $69,35,58,51,26,61)$. Note that $H_{1}$ and $H_{2}$ have no blocks in common. It is therefore possible to trade both $H_{1}$ for $H_{1}^{\prime}=(60,17,44,66,48,24,68,34,57,50$, $25,13)$ and $H_{2}$ for $H_{2}^{\prime}=(61,18,45,67,49,25,69,35,58,51,26,14)$ and obtain a new $\mathrm{S}(5,6,72)$, which we will call $S^{\prime}$. We will prove that $S^{\prime}$ is rigid.

Every hyperquadrilateral in $S$ lies in an orbit of hyperquadrilaterals under $\operatorname{PSL}(2,71)$. Each such orbit gives rise to quadrilaterals (Pasch configurations) in the derived $\mathrm{S}(2,3,69)$ through the points $\infty, 0,1$. Consequently, by examining the quadrilaterals in the derived system and determining which of these extend to hyperquadrilaterals in $S$, it is easily possible to find all the hyperquadrilaterals in $S$ and to determine their signatures. We find that the signatures are of just two types: $(30,0,2)$ and $(12,0,20)$, and that $\operatorname{sig}\left(H_{1}, S\right)=\operatorname{sig}\left(H_{2}, S\right)=$ $(30,0,2)$. We also find that the signatures of $H_{1}^{\prime}$ and $H_{2}^{\prime}$ in $S^{\prime}$ are given by $\operatorname{sig}\left(H_{1}^{\prime}, S^{\prime}\right)=\operatorname{sig}\left(H_{2}^{\prime}, S^{\prime}\right)=(32,0,0)$. This implies that in forming $S^{\prime}$ from $S$, some hyperquadrilaterals are destroyed, but no new ones are formed.

The hyperquadrilaterals of $S$ may be categorized as follows.

Type 1: $H_{1}$ and $H_{2}$.

Type 2: Hyperquadrilaterals other than $H_{1}$ and $H_{2}$ that have a block in common with $\mathrm{H}_{1}$ or $\mathrm{H}_{2}$.

Type 3: Hyperquadrilaterals, other than Types 1 and 2, that have a block in common with a Type 2 hyperquadrilateral.

Type 4: All remaining hyperquadrilaterals.

When $S^{\prime}$ is formed from $S$, Type 1 hyperquadrilaterals are replaced by $H_{1}^{\prime}$ and $H_{2}^{\prime}$, Type 2 hyperquadrilaterals are destroyed, Type 3 hyperquadrilaterals remain but have their signatures altered, and Type 4 hyperquadrilaterals also remain but with their signatures unaltered.

If $H$ is any Type 3 hyperquadrilateral, then we find by computation that $\operatorname{sig}\left(H, S^{\prime}\right)=(30,1,1),(30,2,0),(12,1,19)$ or $(12,2,18)$. Therefore the only hyperquadrilaterals in $S^{\prime}$ that have signature $(32,0,0)$ are $H_{1}^{\prime}$ and $H_{2}^{\prime}$. Consequently any automorphism of $S^{\prime}$ must either map $H_{1}^{\prime}$ to $H_{1}^{\prime}$ and $H_{2}^{\prime}$ to $H_{2}^{\prime}$, or $H_{1}^{\prime}$ to $H_{2}^{\prime}$ and vice-versa.

Now consider the general question of mapping a hyperquadrilateral, $Q_{1}$, to another, $Q_{2}$, in a Steiner system $\mathrm{S}(5,6, v)=T$. The image of the first block of $Q_{1}$ can be any one of the 32 blocks of $Q_{2}$ and the mapping of the two blocks can be done in 6 ! ways. However, having made one of these $32 \times 6 !=23040$ choices, it is not difficult to see that the images of the remaining 6 points of $Q_{1}$ are determined. There are, therefore, just 23040 possible mappings $\phi$ of $Q_{1}$ to $Q_{2}$, and each of these specifies the images of 12 points of $T$. But these 12 points generate $\left(\begin{array}{c}12 \\ 5\end{array}\right)=7925$-tuples, of which only $32 \times 6=192$ appear in the blocks of $Q_{1}$. The remaining 600 5-tuples appear elsewhere amongst the blocks of $T$ and may be used to try to extend the mapping $\phi$ to some, possibly all, of the remaining points of $T$.

For $v=72$ there is a set of 60 blocks of $S^{\prime}$, each of which contains a 5 -tuple from the foundation of $H_{1}^{\prime}$ together with a sixth point outside this foundation, and which are such that these 60 additional points cover all the remaining points 
of $S^{\prime}$. Thus any mapping $\phi$ of $H_{1}^{\prime}$ to itself or to $H_{2}^{\prime}$ can be extended in at most one way to the whole of $S^{\prime}$. Of course, the mapping obtained from these 60 blocks may not map the whole of $S^{\prime}$ to $S^{\prime}$. In fact, it was found that the only mapping which maps $H_{1}^{\prime}$ to itself and preserves $S^{\prime}$ is the identity, and there are no mappings that map $H_{1}^{\prime}$ to $H_{2}^{\prime}$ and preserve $S^{\prime}$. It follows that $S^{\prime}$ is rigid.

\section{$4 v=84$}

We proceed in a similar fashion to the $v=72$ case. Put $G_{83}=\langle\alpha, \beta\rangle$ where $\alpha$ is as before, but now $\beta: z \mapsto \frac{z+1}{z-1}$. Since $-2=9^{2}$ in $\operatorname{GF}(83), G_{83}$ forms an order 4 subgroup of $\operatorname{PSL}(2,83)$. We consider possible hyperquadrilaterals of the same form as described in the previous section, and again the 32 blocks of such a hyperquadrilateral will lie in at most ten orbits under $\operatorname{PSL}(2,83)$. It turns out that, up to equivalence, there are just two choices for the triple $(a, b, c)$ which do not lead to unsuitable or incompatible orbits of 6-tuples. One of the two is given by $(a, b, c)=(5,7,9)$. The corresponding hyperquadrilateral is $H_{1}=(5,7,9,22,29,43,27,20,49,46,71,33)$. The blocks of $H_{1}$ lie in just five orbits under $\operatorname{PSL}(2,83)$. Numerically lowest starters for these orbits are $\{\infty, 0,1,2,9,32\},\{\infty, 0,1,2,12,16\},\{\infty, 0,1,3,24,69\},\{\infty, 0,1,4,25,51\}$ and $\{\infty, 0,1,17,18,44\}$. The last of these gives a sixth orbit, and the others give half orbits.

A computer search gave $12027 \mathrm{~S}(5,6,84)$ systems formed from $\operatorname{PSL}(2,83)$ orbits that include the five orbits specified above. One of these systems has the starters (with $\infty, 0,1$ suppressed for brevity) shown in Table 3 .

$\begin{array}{lrrrr}\{2,9,32\}, & \{2,12,16\}, & \{3,24,69\}, & \{4,25,51\}, & \{17,18,44\}, \\ \{2,3,43\}, & \{2,5,79\}, & \{2,6,34\}, & \{2,7,10\}, & \{2,8,18\}, \\ \{2,11,77\}, & \{2,15,49\}, & \{2,22,27\}, & \{2,31,48\}, & \{2,33,73\}, \\ \{2,35,60\}, & \{3,4,74\}, & \{3,7,51\}, & \{3,8,14\}, & \{3,10,50\}, \\ \{3,15,45\}, & \{3,16,21\}, & \{3,17,38\}, & \{3,18,73\}, & \{3,19,62\}, \\ \{3,25,48\}, & \{3,26,68\}, & \{4,15,66\}, & \{4,20,38\}, & \{4,40,52\}, \\ \{5,8,36\}, & \{5,16,47\}, & \{6,15,35\} . & \end{array}$

Table 3. Starters for an $\mathrm{S}(5,6,84)$.

We will refer to the above system as $S$; it contains $H_{1}$ and $H_{2}=(6,8,10,23$, $30,44,28,21,50,47,72,34)$ which have no blocks in common. It is therefore possible to trade both $H_{1}$ for $H_{1}^{\prime}=(33,7,9,22,29,43,27,20,49,46,71,5)$ and $H_{2}$ for $H_{2}^{\prime}=(34,8,10,23,30,44,28,21,50,47,72,6)$ and obtain a new $\mathrm{S}(5,6,84)$, which we will call $S^{\prime}$ and which we prove is rigid.

The signature of every hyperquadrilateral in $S$ is $(20,12,0)$, and $\operatorname{sig}\left(H_{1}^{\prime}, S^{\prime}\right)=$ $\operatorname{sig}\left(H_{2}^{\prime}, S^{\prime}\right)=(32,0,0)$. Hence in forming $S^{\prime}$ from $S$, some hyperquadrilaterals are destroyed, but no new ones are formed. The hyperquadrilaterals of $S$ may be categorized into the same four Types as in the $v=72$ case. If $H$ is any Type 3 hyperquadrilateral, then we find by computation that $\operatorname{sig}\left(H, S^{\prime}\right)=(21,11,0)$. 
Therefore the only hyperquadrilaterals in $S^{\prime}$ that have signature $(32,0,0)$ are $H_{1}^{\prime}$ and $H_{2}^{\prime}$. Consequently any automorphism of $S^{\prime}$ must either map $H_{1}^{\prime}$ to $H_{1}^{\prime}$ and $H_{2}^{\prime}$ to $H_{2}^{\prime}$, or $H_{1}^{\prime}$ to $H_{2}^{\prime}$ and vice-versa. Arguing as in the previous case, we find a set of 72 blocks of $S^{\prime}$, each of which contains a 5-tuple from the foundation of $H_{1}^{\prime}$ together with a sixth point outside this foundation, and which are such that these 72 additional points cover all the remaining points of $S^{\prime}$. Thus each of the $2 \times 23040$ mappings $\phi$ of $H_{1}^{\prime}$ to itself or to $H_{2}^{\prime}$ can be extended in at most one way to the whole of $S^{\prime}$. In fact, it was found that the only mapping which maps $H_{1}^{\prime}$ to itself and preserves $S^{\prime}$ is the identity, and there are no mappings that map $H_{1}^{\prime}$ to $H_{2}^{\prime}$ and preserve $S^{\prime}$. It follows that $S^{\prime}$ is rigid.

\section{$5 v=108$}

We proceed in a similar fashion to the previous cases. Put $G_{107}=\langle\alpha, \beta\rangle$ where $\alpha, \beta$ are as in the $v=84$ case. Since $-2=31^{2}$ in $\mathrm{GF}(107), G_{107}$ forms an order 4 subgroup of $\operatorname{PSL}(2,107)$. We consider possible hyperquadrilaterals of the same form as those described in the previous sections, and again the 32 blocks of such a hyperquadrilateral will lie in at most ten orbits under $\operatorname{PSL}(2,107)$. Up to equivalence, there are 28 choices for the triple $(a, b, c)$ which do not lead to unsuitable or incompatible orbits of 6 -tuples. One of these is given by $(a, b, c)=(21,43,46)$. The corresponding hyperquadrilateral is $H_{1}=(21,43,46,70,52,76,38,72,81,100,102,56)$. The blocks of $H_{1}$ lie in just five orbits under $\operatorname{PSL}(2,107)$. Numerically lowest starters for these orbits are $\{\infty, 0,1,2,16,49\},\{\infty, 0,1,4,17,61\},\{\infty, 0,1,4,68,84\},\{\infty, 0,1,6,14,84\}$ and $\{\infty, 0,1,7,31,35\}$. The first of these gives a full orbit, the second and third give half orbits, and the fourth and fifth give sixth orbits.

A computer search for $\mathrm{S}(5,6,108)$ systems formed from $\operatorname{PSL}(2,107)$ orbits that include the five orbits specified above was restricted to completions with additional short orbits because it was anticipated that a full search would be both unnecessary and excessively lengthy. There are 466 suitable short orbits of 6 -tuples out of a total of 3242 suitable orbits of 6 -tuples. This heavily restricted search gave 65 solutions, and one of the systems obtained has the starters (with $\infty, 0,1$ suppressed for brevity) shown in Table 4 . We will refer to this system as $S$; it contains $H_{1}$ and $H_{2}=(22,44,47,71,53,77,39,73,82,101,103,57)$ which have no blocks in common. It is therefore possible to trade both $H_{1}$ for $H_{1}^{\prime}=$ $(56,43,46,70,52,76,38,72,81,100,102,21)$ and $H_{2}$ for $H_{2}^{\prime}=(57,44,47,71,53$, $77,39,73,82,101,103,22)$ and obtain a new $\mathrm{S}(5,6,108)$, which we will call $S^{\prime}$ and which we prove is rigid.

The signature of every hyperquadrilateral in $S$ is $(26,0,6)$, and $\operatorname{sig}\left(H_{1}^{\prime}, S^{\prime}\right)=$ $\operatorname{sig}\left(H_{2}^{\prime}, S^{\prime}\right)=(32,0,0)$. Hence in forming $S^{\prime}$ from $S$, some hyperquadrilaterals are destroyed, but no new ones are formed. The hyperquadrilaterals of $S$ may be categorized into the same four Types as in the $v=72$ case. If $H$ is any Type 3 hyperquadrilateral, then we find by computation that $\operatorname{sig}\left(H, S^{\prime}\right)=(26,1,5)$ or $(26,2,4)$. Therefore the only hyperquadrilaterals in $S^{\prime}$ that have signature $(32,0,0)$ are $H_{1}^{\prime}$ and $H_{2}^{\prime}$. Consequently any automorphism of $S^{\prime}$ must either 


$\begin{array}{ccccc}\{2,16,49\} & \{4,17,61\} & \{4,68,84\} & \{6,14,84\} & \{7,31,35\} \\ \{2,3,104\} & \{2,5,81\} & \{2,6,36\} & \{2,7,102\} & \{2,8,38\} \\ \{2,9,68\} & \{2,10,20\} & \{2,11,33\} & \{2,12,83\} & \{2,13,61\} \\ \{2,22,52\} & \{2,25,60\} & \{2,34,63\} & \{2,40,91\} & \{2,47,82\} \\ \{3,4,9\} & \{3,7,87\} & \{3,8,24\} & \{3,10,90\} & \{3,11,76\} \\ \{3,12,75\} & \{3,14,16\} & \{3,17,74\} & \{3,19,64\} & \{3,20,70\} \\ \{3,27,61\} & \{3,29,56\} & \{3,31,48\} & \{3,32,96\} & \{3,41,80\} \\ \{3,42,105\} & \{3,44,71\} & \{3,45,86\} & \{3,88,100\} & \{4,5,92\} \\ \{4,11,35\} & \{4,15,39\} & \{4,20,50\} & \{4,21,32\} & \{4,24,94\} \\ \{4,28,83\} & \{4,31,33\} & \{4,38,45\} & \{4,46,60\} & \{4,47,57\} \\ \{5,6,25\} & \{5,8,31\} & \{5,24,76\} & \{5,26,95\} & \{5,49,94\} \\ \{6,13,91\} & \{6,30,70\} & \{6,33,78\} & \{6,39,60\} & \{7,8,68\} \\ \{7,23,85\} & \{9,20,73\} & \{9,30,99\} & & \end{array}$

Table 4. Starters for an $\mathrm{S}(5,6,108)$.

map $H_{1}^{\prime}$ to $H_{1}^{\prime}$ and $H_{2}^{\prime}$ to $H_{2}^{\prime}$, or $H_{1}^{\prime}$ to $H_{2}^{\prime}$ and vice-versa. Arguing as in the previous cases, we find a set of 96 blocks of $S^{\prime}$, each of which contains a 5-tuple from the foundation of $H_{1}^{\prime}$ together with a sixth point outside this foundation, and which are such that these 96 additional points cover all the remaining points of $S^{\prime}$. Thus each of the $2 \times 23040$ mappings $\phi$ of $H_{1}^{\prime}$ to itself or to $H_{2}^{\prime}$ can be extended in at most one way to the whole of $S^{\prime}$. It was found that the only mapping which maps $H_{1}^{\prime}$ to itself and preserves $S^{\prime}$ is the identity, and there are no mappings that map $H_{1}^{\prime}$ to $H_{2}^{\prime}$ and preserve $S^{\prime}$. It follows that $S^{\prime}$ is rigid.

\section{Concluding remarks}

All the computations and searches described in this paper were done on an ordinary home PC; to be precise, a $2.4 \mathrm{GHz}$ Pentium $4 \mathrm{C}$ with 512MB RAM and using a "Windows XP Professional" operating system. In each case, the systems $\mathrm{S}(5,6, v)$ were determined by listing each 6 -block orbit with the 5 -block orbits which it contains, and each 5-block orbit with the 6-block orbits which contain it. A backtracking procedure was then used to identify packings of all the 5-block orbits into 6 -block orbits. The longest computation, determining the 12027 systems for $v=84$, took around 200 hours.

Although we have only presented three rigid $\mathrm{S}(5,6, v)$ systems, it is clear that the method is capable of producing a large number of new systems for $v=72,84$ and 108 . There is also little doubt that many larger $S(5,6, v)$ systems will contain hyperquadrilateral trades, and that applying such trades will often result in systems that do not have PSL automorphism groups. We have used a particular form for our hyperquadrilaterals and other forms may be equally effective. Indeed, there may be other trades that can be used both for these systems and other large systems such as $t-(v, k, \lambda)$ designs with $k \geq 4$. 


\section{References}

[1] R. J. R. Abel and M. Greig, BIBDs with small block size, in: The Handbook of Combinatorial Designs, second edition, C. J. Colbourn and J. H. Dinitz (editors), CRC Press, Boca Raton, 2007, 72-79.

[2] A. Betten, R. Laue, S. Molodtsov and A. Wassermann, Steiner systems with automorphism groups $\operatorname{PSL}(2,71)$, PSL $(2,83)$, and P $\Sigma L\left(2,3^{5}\right)$, J. Geom., $67,2000,35-41$.

[3] A. Betten, R. Laue and A. Wassermann, A Steiner 5-design on 36 points, Des. Codes Crypt., 17, 1999, 181-186.

[4] C. J. Colbourn and A. Rosa, Triple Systems, Clarendon Press, New York, 1999.

[5] R. H. F. Denniston, Some new 5-designs, Bull. London Math. Soc., 8, 1976, 263-267.

[6] M. J. Grannell and T. S. Griggs, A note on the Steiner systems S(5, 6, 24), Ars Combin., 8, 1979, 45-48.

[7] M. J. Grannell, T. S. Griggs and R. A. Mathon, On Steiner systems S(5, 6, 48), J. Combin. Math. Combin. Comput., 12, 1992, 77-96.

[8] M. J. Grannell, T. S. Griggs and R. A. Mathon, Some Steiner 5-designs with 108 and 132 points, J. Combin. Des., 1, 1993, 213-238.

[9] M. J. Grannell, T. S. Griggs and R. A. Mathon, Steiner systems $\mathrm{S}(5,6, v)$ with $v=72$ and 84, Math. Comp., 67, 1998, 357-359.

[10] A. Hartman and K. T. Phelps, Steiner quadruple systems, in: Contemporary Design Theory, J. H. Dinitz and D. R. Stinson (editors), Wiley, 1992, 205-240.

[11] G. B. Khosrovshahi and R. Laue, $t$-Designs with $t \geq 3$, in: The Handbook of Combinatorial Designs, second edition, C. J. Colbourn and J. H. Dinitz (editors), CRC Press, Boca Raton, 2007, 79-101.

[12] T. P. Kirkman, On a problem in combinations, Cambridge and Dublin Math. J., 2, 1847, 191-204.

[13] W. H. Mills, A new 5-design, Ars Combin., 6, 1978, 193-195.

[14] E. Witt, Die 5-fach transitiven Gruppen von Mathieu, Abh. Math. Sem. Univ. Hamburg, 12, 1938, 256-264. 